\title{
An Interprofessional Approach to Teaching Evidence-based Practice
}

Jennifer L. Gresham - Anderson

Midwestern State University, jennifer.anderson@msutexas.edu

Kathleen M. Williamson

Midwestern State University, kathleen.williamson@msutexas.edu

Satyanarayana Ayinagadda

Midwestern State University, satya.ayinagadda@msutexas.edu

Follow this and additional works at: https://nsuworks.nova.edu/ijahsp

Part of the Medicine and Health Sciences Commons

\section{Recommended Citation}

Gresham - Anderson JL, Williamson KM, Ayinagadda S. An Interprofessional Approach to Teaching Evidence-based Practice. The Internet Journal of Allied Health Sciences and Practice. 2020 Jan 01;18(1), Article 2.

This Manuscript is brought to you for free and open access by the College of Health Care Sciences at NSUWorks. It has been accepted for inclusion in Internet Journal of Allied Health Sciences and Practice by an authorized editor of NSUWorks. For more information, please contact nsuworks@nova.edu. 


\title{
An Interprofessional Approach to Teaching Evidence-based Practice
}

\begin{abstract}
Purpose: The purpose of this mixed methods study was to identify students' engagement and implementation of EBP competencies after exposure to EBP content in an interprofessional online research course. In this research, a diverse, convenience sample of nursing, respiratory care, and social work students were selected. The results aim to assist educators in preparing the next generation for their first professional role in providing care using the best evidence. The research questions that guided the study were: (1) What are the levels of practice, attitude, knowledge and skill regarding EBP among the sample? (2) What are the barriers and facilitators to acquiring EBP competency? (3) What are the differences in EBP attitudes, knowledge, and skills between the diverse disciplines in the sample? Methods: This descriptive mixed methods study employed a cross sectional design that allowed comparisons of programs and the different levels within the program. Quantitative data was collected using the SEBPQ survey, allowing participants' the opportunity to self-report on the practice, attitude, and knowledge/ skills. Qualitative data was collected through two focus groups of approximately six to ten students from the three programs. Results: Data showed that students within the three selected disciplines had a positive attitude towards EBP. Theme analysis supported this view as participants noted EBP impacts patient safety and quality care. Barriers identified included, "lack of resources, use of research language interchangeably with EBP, quick exposure to EBP and confusion among EBP practices in the clinical setting." Participants had an awareness of EBP and recognized the value of EBP. However, there may be ways to improve the implementation of EBP throughout the learning process while students are in undergraduate studies. Conclusion: Using an interprofessional online research course to introduce students to EBP was successful. Providing an interprofessional approach to teaching EBP will provide support the implementation of EBP competencies. Further development of additional interprofessional EBP courses or activities should be considered. This study found that if interprofessional care teams' work together and use the EBP process in their courses it will improve student perceived value of EBP.
\end{abstract}

\section{Author Bio(s)}

Jennifer L. Gresham-Anderson, EdD, RRT, RRT-NPS, is an Assistant Professor and Chair of the Respiratory Care Program in the Robert D. \& Carol Gunn College of Health Sciences \& Human Serves at Midwestern State University in Wichita Falls, TX.

Kathleen M. Williamson, MSN, Ph.D., RN served as Professor and Chair of the Wilson School of Nursing in the Robert D. \& Carol Gunn College of Health Sciences \& Human Serves at Midwestern State University in Wichita Falls, TX from 2015 - 2018.

Satya N. Ayinagadda, MSW, Ph.D., is an Associate Professor and Chair of the Social Work Department in the Robert D. \& Carol Gunn College of Health Sciences \& Human Serves at Midwestern State University in Wichita Falls, TX. 


\title{
IIJAHSP ${ }^{m}$ \\ The Internet Journal of Allied Health Sciences and Practice \\ Dedicated to allied health professional practice and education
}

Vol. 18 No. 1 ISSN 1540-580X

\section{An Interprofessional Approach to Teaching Evidence-based Practice}

\author{
Jennifer L. Gresham-Anderson \\ Kathleen M. Williamson \\ Satyanarayana Ayinagadda \\ Midwestern University \\ United States
}

\begin{abstract}
Purpose: The purpose of this mixed methods study was to identify students' engagement and implementation of EBP competencies after exposure to EBP content in an interprofessional online research course. In this research, a diverse, convenience sample of nursing, respiratory care, and social work students were selected. The results aim to assist educators in preparing the next generation for their first professional role in providing care using the best evidence. The research questions that guided the study were: (1) What are the levels of practice, attitude, knowledge and skill regarding EBP among the sample? (2) What are the barriers and facilitators to acquiring EBP competency? (3) What are the differences in EBP attitudes, knowledge, and skills between the diverse disciplines in the sample? Methods: This descriptive mixed methods study employed a cross sectional design that allowed comparisons of programs and the different levels within the program. Quantitative data was collected using the S-EBPQ survey, allowing participants' the opportunity to self-report on the practice, attitude, and knowledge/skills. Qualitative data was collected through two focus groups of approximately six to ten students from the three programs. Results: Data showed that students within the three selected disciplines had a positive attitude towards EBP. Theme analysis supported this view as participants noted EBP impacts patient safety and quality care. Barriers identified included, "lack of resources, use of research language interchangeably with EBP, quick exposure to EBP and confusion among EBP practices in the clinical setting." Participants had an awareness of EBP and recognized the value of EBP. However, there may be ways to improve the implementation of EBP throughout the learning process while students are in undergraduate studies. Conclusion: Using an interprofessional online research course to introduce students to EBP was successful. Providing an interprofessional approach to teaching EBP will provide support the implementation of EBP competencies. Further development of additional interprofessional EBP courses or activities should be considered. This study found that if interprofessional care teams' work together and use the EBP process in their courses it will improve student perceived value of EBP.
\end{abstract}

Keywords: Evidence-based Practice, Interprofessional, Nursing, Social Work, Respiratory Care, Mixed Methods 


\section{INTRODUCTION}

Evidence based practice (EBP) provides a scientific frame of reference to help determine the best solution for important inquiries about health care. The development of practice models firmly rooted in clinical research increases the effectiveness of health services. All stakeholders tend to benefit through improved quality of care, increased transparency, and reduced costs. EBP encourages clinical decisions based on current evidence, patient values, and professional expertise supplanting other models that may be based on tradition, instinct, or advice given by authority. Awareness of EBP should begin early in the training of health care providers. ${ }^{1}$ Students often find difficulty in translating the knowledge acquired in the classroom into effective clinical practice. Moreover, veteran educators may have little experience in creating curricula that implements EBP concepts. Current evidence suggests among nursing personnel, EBP has not been fully integrated into clinical practice. ${ }^{2-6}$ Evaluation of current educational models designed to implement EBP concepts are necessary if educators are to assure educational initiatives are effective.

This study was conducted at a public liberal arts university located in the mid-west region of the United States. The university has a large health science and human service college that represents a third of the campus student population. An interprofessional approach to teaching EBP within the college was implemented five years ago. An online interprofessional research course was added as a degree requirement for respiratory care, social work, nursing, and radiology. The interprofessional research course introduces students within the college to EBP. EBP is integrated into several respiratory care courses. Nursing students also have an EBP lesson in their leadership course. EBP is included in social work practice courses that focus on working with families, groups, and communities. Students in all three disciplines are exposed to EBP in the clinical practicum settings. The study was limited to three programs within the college (respiratory care, social work, and nursing) because these programs have specific accreditation requirements to include EBP in the curriculum. The study was needed to determine if an online interprofessional approach to teaching EBP, along with individual program specific EBP curriculum was effective. The goal of the study was to assess the practices, attitudes, and knowledge/skills of EBP in health careers students to improve pedagogical practices to fully support learning the EBP process.

\section{LITERATURE REVIEW}

Research has shown that education is the most influential in support of implementing the EBP process in practice especially before students transition to formal careers. 5,7 Undergraduate health care students need to be educated and conditioned to adopt the skills that are necessary to provide safe practice. Due to the lack of research to support the ideal curriculum/course sequence that supports learning the EBP paradigm and competencies, it is important to assess the attitudes, skills and abilities of undergraduate students. ${ }^{8}$ Knowing the students' competencies, educators will be able to correct or improve pedagogical practices to fully support students transition into their careers. Barriers include, lack of resources, interchanging EBP and research language, lack of exposure to EBP and confusion among EBP practices in the clinical setting. Finding ways to remove the barriers in academic settings will reduce the different practices. ${ }^{5,9}$ In interprofessional environments, the language, model and approaches to EBP may differ due to differences in training and health disciplines. This can act as a barrier in coordination and communication in instances where an interprofessional team is necessary for a patient's treatment. Each discipline can contribute greatly to the implementation of EBP but research development and training has primarily been specific to each discipline..$^{10}$

The research shows that bachelor level students do not read research articles. ${ }^{11}$ This is due to the fact that they find it hard to understand the research and are not eager to use the information. ${ }^{12}$ Indications point to a lack of research skills and uptake by nursing, respiratory therapists and social workers. ${ }^{13-14}$ Research studies have identified inconsistencies in the adoption and implementation of EBP in the workplace and in educational settings. ${ }^{15-16}$ Additionally, research suggests that healthcare providers may struggle to implement EBP even after they have learned the skills through in-services and other educational practices $5,12,17$. A variety of reasons such as patchy uptake of EBP, lack of confidence and fear of making mistakes, and understanding how research impacts practice and resource demands. ${ }^{17-21}$

Developing healthcare provider competencies in EBP are essential in today's healthcare setting. Education appears to be the most influential force for the application and execution of EBP competencies. If undergraduate students are educated and conditioned to adopt the skills and competencies necessary, and molded to develop an inspired and motivated attitude, the barriers to EBP implementation will be reduced in the different practices.3,5,9 It is important to assess the practices, attitudes, and knowledge/skills of EBP in health career students in an effort to improve pedagogical practices to fully support learning the EBP process.

\section{PURPOSE}

There is a lack of research on the effectiveness of EBP teaching across various health care disciplines, such as respiratory care. The purpose of this study was to identify students' engagement and implementation of EBP competencies after exposure to EBP content in an online course. ${ }^{22}$ In this research, a diverse, convenience sample of nursing, respiratory care, and social work students 
were selected. The study was designed to evaluate the effectiveness of EBP introduction in an online interprofessional research course consisting of a diverse group of undergraduate health career students. The research questions that guided the study were: (1) What are the levels of practice, attitude, knowledge and skill regarding EBP among the sample? (2) What are the barriers and facilitators to acquiring EBP competency? (3) What are the differences in EBP attitudes, knowledge, and skills between the diverse disciplines in the sample?

\section{METHODS \\ Design}

This descriptive mixed methods study employed a cross sectional design that allowed comparison of programs and the different levels of EBP understanding within the programs. An online interprofessional research course was added as a degree requirement for respiratory care, social work, nursing, and radiology. The interprofessional research course introduces students within the college to EBP in module one. Students must read a textbook chapter, articles, and review a lecture on EBP. Additional course content includes a combination of lectures, readings, and assignments designed to assist learners to understand why and how different professional disciplines must work together to generate and disseminate knowledge.

The tool utilized was the Student Evidence-based Practice Questionnaire (S-EBPQ) developed by Upton, Scurlock-Evans, \& Upton. ${ }^{22}$ The S-EBPQ is a 21 -item survey comprised of four subscales to measure 1) Practice - Frequency of Practice [7 items], 2) Attitude towards EBP [3 items], 3) Retrieving and Reviewing the Evidence [7 items], and 4) Sharing and Applying EBP [7 items] all items were on a 1-7 Likert Scale.22-23 This tool was specifically developed to facilitate "meaningful comparisons between the understanding and application of EBP at all levels of practice from student through newly qualified practitioner to established specialist nurse practitioner."22

Quantitative data was collected using the S-EBPQ survey, allowing participants' the opportunity to self-report on the practice, attitude, and knowledge/skills of EBP. The survey was distributed to Junior and Senior students who completed the interprofessional research course. Students complete the course at various times, as it is a prerequisite course. The instrument is quick and easy to administer and has been found to possess both construct and discriminant validity; internal reliability estimates are reported as good. According to Upton, Scurlock-Evans and Upton, "the S-EBPQ appears to be a psychometrically robust measure of EBP practice, attitudes, and knowledge and skills."22 The Cronbach's a ranges from 0.765 to 0.912 for the subscales. ${ }^{22}$ The Cronbach's a for this study was 0.92 . The questionnaire includes two factors: retrieving and reviewing evidence and sharing and applying EBP. According to Upton, Scurlock-Evan, and Upton, "they represent a refinement of the EBPQ knowledge/skill subscale to differentiate between aspects of EBP relating to evidence identification, evaluation, and the application of the EBP process and sharing knowledge."22 The survey tool also collected basic demographic and program information. For the Respiratory Care undergraduate students in the online program, responses were collected via a questionnaire hosted on Survey Monkey@. Aell others received a paper copy to complete.

Qualitative data was collected through two focus groups of approximately six to ten students from the three programs. The authors tried to get input from students in all three professional programs. Students who participated in the focus group had taken the online interprofessional research course. A standard protocol was used for each focus group (see Appendix A). Participants were informed they could leave at any time, that their identity would be kept confidential, and responses to any of the questions will not be reported in a way that could reveal who they are. The focus groups were conducted by a moderator and a researcher. In both focus groups students openly, discussed questions in relation to the EBP and research processes. Each focus group ran approximately 30 to 60 minutes. During each focus group discussion, the moderator transcribed answers for each open-ended question. Participants were able to check the accuracy of the information written for verification. Researchers also took field notes during each focus group and identified common themes after saturation was achieved through an analytic inductive analysis

\section{Participants and Setting}

The target population for this study was a convenience sample of the authors' undergraduate nursing students ( $N=350)$, respiratory care students $(\mathrm{N}=147)$, and social work students $(\mathrm{N}=50)$ at a public university located in the mid-west region of the United States. The students were males and females between the ages of 18-64. The cross-section of groups identified were nursing (Accelerated-RN and Pre-licensure first - fifth semester), respiratory care (on campus [juniors and seniors] \& online [completion program]), and social work (juniors \& seniors). Students from the three programs who participated were studying in programs located in the College of Health Sciences and Human Services. Participants in the focus groups ages ranged from 21 to 28 years

(C) The Internet Journal of Allied Health Sciences and Practice, 2019 
old, ten were female and five were male. Eight participants were respiratory care students, three were nursing students and four were social work students. Students were invited to participate in one of two focus groups. Researchers went to courses and invited students to attend the focus groups. Flyers with information including the date and time of the two focus groups were hung throughout the health science building. Students were informed that participation was voluntary, and that they could quit at any time, and there would be no effect upon their grades or relationship with their program and/or the university. They were also informed that a summary of themes and findings would be available upon request. Participants gave their time willingly and seemed interested in participating, regardless of the lack of incentives or compensation.

\section{Ethical Considerations}

The study, 17012701 was approved by the Institutional Review Board at the university. A cover letter was read to all students who participated in the study, implied consent was established when the subject/participants completed the demographic and EBP student survey and/or attended a focus group session. There was no compensation to participants. The survey tool was structured to allow anonymity at the level of data analysis and reporting in aggregate, with no subject-identifying information. The information provided by the participants was treated confidentially. Data was secured in password protected software and in password protected computers.

\section{Data Collection and Analysis}

Access to potential participants was granted through faculty in the College of Health Science and Human Services. Researchers went into classes and presented students the opportunity to participate in the study. Students were presented with survey documents at the end of strategically selected classes and informed of their rights. An electronic duplicate of the survey was disseminated (using Survey MonkeyC) to students with primarily online presence. If more than $10 \%$ of the questions on a survey were not completed, the survey was eliminated and immediately deleted/destroyed.

Quantitative data was inputted into IBM SPSS Statistics 22. Confidence internals were set at $95 \%$, and $p<.05$ was deemed significant. Any statistical significance as determined by the omnibus p-value from the ANOVA was post-hoc tested using the Tukey procedure. This test was used to determine where specific group differences exist. The measures of central tendency and measures of dispersion of items on the Demographic Data Questionnaire were computed.

Qualitative data analysis allowed for comparisons between the sample and identified groups. Participation was voluntary for the focus groups. Researchers made signs to inform students about participating in the focus groups. Faculty from each discipline offered extra credit points for students who volunteered to participate in focus groups. The data collected during the focus group was anonymous and coded to protect the participants. Responses were collected from two focus groups of 6-10 students. The 3060 -minute discussions were guided by open-ended and semi-structured questions to encourage open dialogue. Focus groups were conducted by researchers from nursing, respiratory care, and an undergraduate respiratory care student. Prior to the focus group researchers and the undergraduate student discussed the focus group protocol and how to conduct the focus groups. There was a moderator and a researcher in each group. To eliminate bias researchers did not conduct focus groups with students from their discipline. Researchers identified common themes after saturation was achieved through an analytic inductive analysis. To ensure credibility of the data and consistency of themes generated, a debriefing with the researchers was conducted to verify themes formed during the data analysis. Throughout the data collection process, the researchers responded to the correspondents of the participants and tried to obtain detail to provide an enhanced understanding of their responses. The data-gathering process continued in each focus group until a continuous feedback loop between data, data analysis, results, and conclusions occurred. The focus group facilitators posted the themes collected from each question on a flip chart and the participants verified the themes.

\section{RESULTS}

\section{Quantitative Data}

The overall response rate was $68 \%(n=372)$. By department, $79 \%$ nursing students $(n=275), 39 \%$ respiratory care students $(n=57)$, and $80 \%$ social work students $(n=40)$ participated. Demographic information indicated that there were 301 females and 71 males between the ages of 19 and 59 . The mean age was 25 and the median age was 23 . Three hundred and thirty- three (89\%) of the respondents have heard of EBP and $33(9 \%)$ were unfamiliar with the term (See Table 1). There were six (2\%) questionnaires with missing responses for this question. The majority of students in all three disciplines were familiar with EBP. 
INTERPROFESSIONAL APPROACH TO TEACHING

Table 1. Participants' Familiarity with the Term EBP

\begin{tabular}{|c|c|}
\hline Program & Participants' Familiarity (\%) \\
\hline Nursing $(251=$ yes; $20=$ no) & $91 \%$ \\
\hline Respiratory Care $(48=$ yes; $8=$ no) & $85 \%$ \\
\hline Social Work $(43=$ yes; $5=$ no) & $85 \%$ \\
\hline
\end{tabular}

For the three programs combined, mean (Standard Deviation) for practice was 4.39 (SD 1.39) (See Tables 2 and 3). The mean for attitude was 5.60 (SD 1.15). The mean for knowledge was 4.70 (SD 0.98). When the knowledge subscale was calculated using two factors, the mean for "retrieving and reviewing evidence" was 4.62 (SD 1.02) and the mean for "sharing and applying EBP" was 4.80 (SD 1.10). The overall mean for the S-EBPQ for this program was 4.74 (SD 0.91).

Table 2. Three Programs Combined Overall Mean for Practice, Attitude, and Knowledge

\begin{tabular}{|l|l|}
\hline S-EBPQ Overall Scores & Mean (Standard Deviation) \\
\hline Practice & 4.39 (SD 1.39) \\
\hline Attitude & 5.60 (SD 1.15) \\
\hline Knowledge/Skills & 4.70 (SD 0.98) \\
\hline Subscale: Retrieving and reviewing evidence & 4.62 (SD 1.02) \\
\hline Subscale: Sharing and applying EBP & 4.80 (SD 1.10) \\
\hline Overall EBP & 4.74 (SD 0.91) \\
\hline
\end{tabular}

Table 3. Programs Overall Mean Scores (Standard Deviations) for each Program by Category and Subscale of S-EBPQ

\begin{tabular}{|c|c|c|c|c|c|c|}
\hline EBP Scales: & Practice & Attitude & Knowledge/Skills & \multicolumn{2}{|c|}{ Knowledge/Skills: Subscales } & $\begin{array}{c}\text { Overall EBF } \\
\text { Score }\end{array}$ \\
\hline Programs: & & & & $\begin{array}{l}\text { * Retrieving and } \\
\text { Reviewing } \\
\text { Evidence }\end{array}$ & $\begin{array}{l}\text { Sharing and } \\
\text { Applying EBP }\end{array}$ & \\
\hline $\begin{array}{l}\text { Respiratory Care } \\
\text { Program }\end{array}$ & $\begin{array}{l}4.78 \\
\text { (SD 1.22) }\end{array}$ & $\begin{array}{l}5.85 \\
(S D 1.01)\end{array}$ & $\begin{array}{l}4.98 \\
(S D 0.87)\end{array}$ & $\begin{array}{l}4.80 \\
(S D 0.97)\end{array}$ & $\begin{array}{l}5.26 \\
\text { (SD 0.89) }\end{array}$ & $\begin{array}{l}5.05 \\
\text { (SD 0.82) }\end{array}$ \\
\hline
\end{tabular}




\begin{tabular}{|l|l|l|l|l|l|l|} 
Social Work & $\begin{array}{l}4.82 \\
\text { Program }\end{array}$ & $\begin{array}{l}5.13 \\
\text { (SD 1.22) }\end{array}$ & $\begin{array}{l}4.08 \\
\text { (SD 1.50) }\end{array}$ & $\begin{array}{l}4.92 \\
\text { (SD 1.00) }\end{array}$ & $\begin{array}{l}5.07 \\
\text { (SD 0.87) }\end{array}$ & (SD 0.82) \\
\hline Nursing Program & $\begin{array}{l}4.25 \\
\text { (SD 1.80) }\end{array}$ & $\begin{array}{l}5.61 \\
\text { (SD 1.56) }\end{array}$ & $\begin{array}{l}4.60 \\
\text { (SD 1.00) }\end{array}$ & $\begin{array}{l}\text { (SD 1.02) } \\
\text { (SD 1.14) }\end{array}$ & (SD 1.01) \\
\hline
\end{tabular}

${ }^{*}$ Not significantly different between programs $(p>0.05)$

One-way ANOVAs with post hoc tests were performed to answer if there were differences in perceived Practice, Attitudes, Knowledge/Skills, Retrieving and Reviewing Evidence, Sharing and Applying EBP and Total EBP among the three programs. There were significant differences in Practice $(F(2,368)=5.551, p=0.004)$; Attitude $(F(2,366)=4.91, p=0.008)$; Knowledge/Skills $(F(2,365)=5.34, p=0.003)$; Retrieving and Reviewing Evidence $(F(2,365)=3.22, p=0.041)$; Sharing and Applying Evidence $(F(2,364)=8.44, p=0.000)$; and Total $\operatorname{EBP}(F(2.368)=6.26, p=0.002)$. Tukey post hoc tests indicated significant differences between the programs within all the categories except for Retrieving and Reviewing Evidence. Retrieving and reviewing evidence is covered in the interprofessional research course.

There were significant differences between the nursing and the respiratory programs in four of the six categories (See Table 4): Practice $(p=0.25)$, Knowledge/Skills $(p=0.16)$, Sharing and Applying $\operatorname{EBP}(p=0.001)$, and Total $\operatorname{EBP}(p=0.005)$. Compared to the students in the nursing program, students in the respiratory care program had higher mean scores in each of the categories.

There were significant differences between the nursing and the social work programs in three of the six categories: Practice $(p=0.041)$, Attitude $(p=0.03)$, and Knowledge/Skills $(p=0.05)$. Students in the social work program had higher mean scores in the categories of Practice and Knowledge/Skills. Students in nursing had a higher mean score in the category of Attitude.

There was a significant difference between the social work and the respiratory programs in Attitude $(p=0.006)$. Students in the respiratory program had a higher mean score than the students in the social work program.

Table 4. Mean Scores (Standard Deviations) of Each Program by Category of EBP

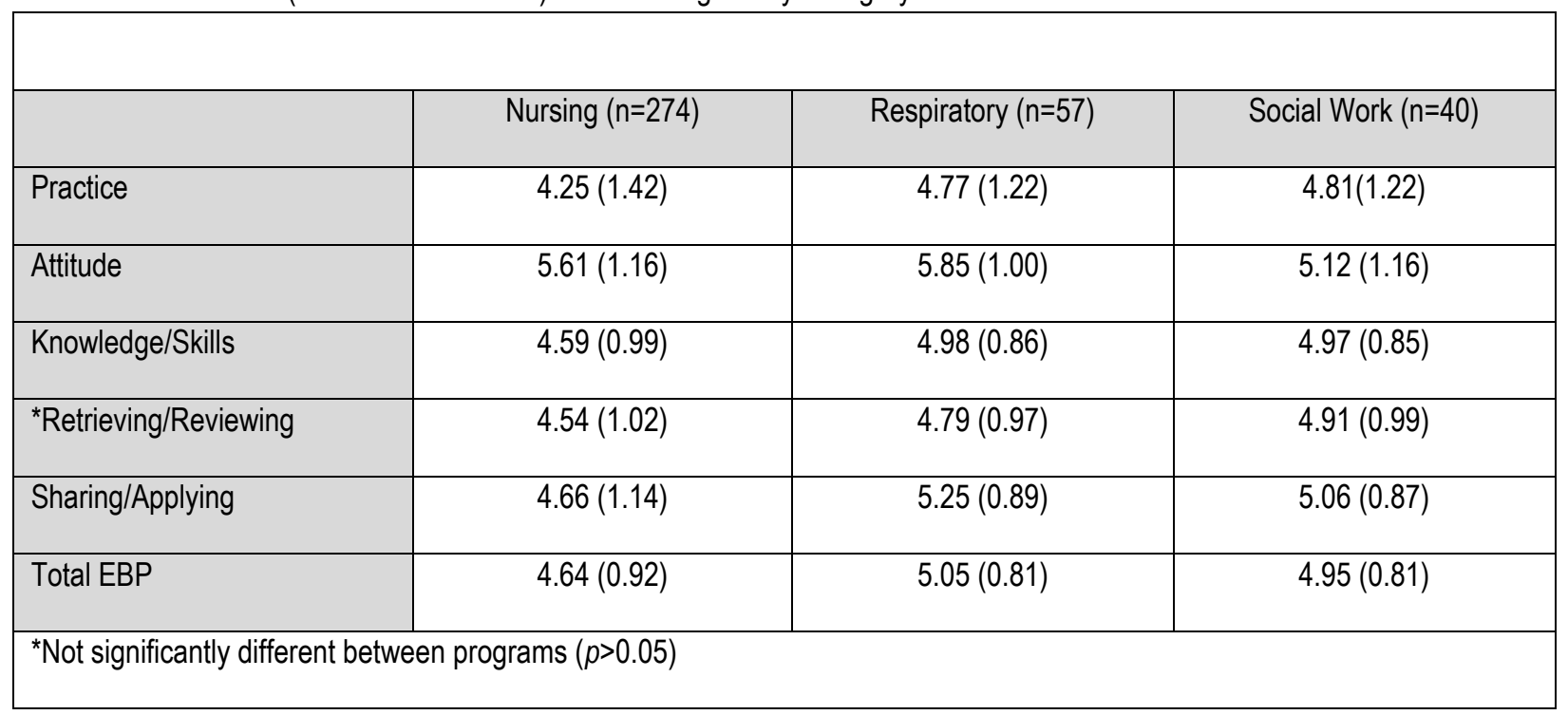

\section{Qualitative Data}

Qualitative data was derived from two focus groups made of 15 students from the three programs. The focus groups were conducted by researchers from nursing, respiratory care, and an undergraduate respiratory care student. To eliminate bias researchers did not conduct focus groups with students from their discipline. Participants ages ranged from 21 to 28 years old, 
ten were female and five were male. Eight participants were respiratory care students, three were nursing students and four were social work students. Most had participated in clinical or observation courses in their major. All participants acknowledged that they had heard of EBP. Students openly discussed questions in relation to EBP, each focus group ran approximately 30 to 60 minutes. Interpretation of emerging themes enabled the researchers to draw meaning from data, leading to a deeper understanding about their practice of, attitudes towards, and knowledge/skills of EBP. The qualitative data from the two focus groups yielded saturation of themes related to EBP. Themes included research, patient safety, quality patient care, and evidence.

\section{Theme 1: Research}

Participants overwhelmingly related EBP with research. Participants frequently commented that EBP is a type of research. Students stated, "there are barriers" such as: "having a lack of knowledge on access to resources", "lack of knowledge on knowing where to look for evidence", and a "lack of knowledge about research and EBP." They also expressed concerns that in their clinical experience there was a lack of current research because many health care workers are "stuck in their own ways."

Representative comments included:

"Research is using secondary data and EBP is collecting data to support research."

"There is a lack of knowledge among health care providers of how to access EBP"

"If research is data, they trust the larger numbers. If EBP is trial and error, is there validity?"

"I have used EBP for a literature review to compare causes of stress among healthcare providers."

"Experts in the community gather and collect data to help families and meet the needs of the community."

\section{Theme 2: Patient Safety}

Participants from all disciplines expressed that EBP was very important and helped to improve patient safety. Participants gave examples about how EBP was used for protocols to improve patient safety related to: CAUTI (Catheter-Associated Urinary Tract Infections), tracheostomy care, and ventilator management.

Representative comments included:

"EBP improves patient safety in the clinical setting"

"EBP is evidence and research from clinical practice that provides guidelines on safety to make improvements in health care" "EBP is thinking about safety in health care"

"EBP helps to create guidelines to promote safe practice"

\section{Theme 3: Quality Patient Care}

During the focus group discussions, another theme that emerged was quality patient care. All participants agreed that EBP was important to improve the quality of patient care.

Representative comments included:

"Based on the findings from research (EBP) you put the findings into practice to improve quality patient care."

"Concrete evidence is better than hearsay and improves patient care."

\section{Theme 4: Evidence}

Participants agreed that EBP provided important evidence for patients and families. They agreed that EBP helps with liability issues.

Representative comments included:

"The more we seek evidence and research the more we keep on the cutting edge of healthcare."

"Lack of resources in research with field of study"

"EBP is important in social work to show families evidence in practice"

"Evidence that shows a practice is effective in the healthcare world is important for patients"

Engaging with students in the focus group allowed researchers to better understand the perceptions of EBP among students.

\section{MIXED METHODOLOGY}

Mixed methods research triangulates results that offer higher validity and reliability. Therefore, researchers compared the qualitative and quantitative data. Both qualitative and quantitative data revealed that participants in all three disciplines recognized the importance of the practice, attitude, and knowledge/skills of EBP. However, it is important to note that there was confusion of 
the terminology and the word EBP and research was interchanged among most participants during the focus groups. Quantitative results revealed that eighty-seven percent $(87 \%)$ of students in the three programs were familiar with EBP. Yet, a small percentage $(9 \%)$ of participants were unfamiliar with the term. Students were less likely to practice critical appraisal (M 4.02) and share information with colleagues (M 4.10). Participants were more likely to retrieve evidence (M 4.96) and share ideas (M 4.96). The differences in perceived Practice, Attitudes, Knowledge/Skills, among the three programs may be due to the differences in clinical practicums and classification level. Each program has different clinical practicum requirements. Students in each program are exposed to different levels of EBP during clinical courses

\section{DISCUSSION}

The researchers found a gap in the literature related to studies using interprofessional research courses to introduce students to EBP. While much has been written about facilitators and barriers of conducting interprofessional research, the curriculum development of interprofessional research courses is rarely mentioned. In the National Academy of Science report, "Facilitating Interdisciplinary Research," for example, the chapter devoted to the academic researcher describes immersion courses and interdisciplinary curricula for undergraduate and graduate students, but does not explicitly recommend what content would be important to develop interprofessional expertise and did not specify mention of EBP. ${ }^{24}$ No articles were found on introducing undergraduate Respiratory Care, Nursing, and Social Work students to EBP.

Respiratory care, social work, and nursing professions have worked to increase EBP knowledge, skills and abilities of individuals within the fields through accreditation processes. American Association for Respiratory Care (AARC) supports the use of therapist implemented protocols as effective tools for producing improved patient outcomes and allocation of services. ${ }^{25}$ According to Barnes, Gale, Kacmarek, and Kageler, "use of evidence-based protocols that follow a nationally accepted standard of practice and application of biomedical innovation continue to be important competency areas for RTs [Respiratory Therapists]." 26 The American Association of Colleges of Nursing essential documents has set prescient in the implementation of EBP into a bachelor's of nursing curriculum. ${ }^{27}$ The Essentials of Baccalaureate Education for Professional Practice, Essential III: Scholarship for Evidence Based Practice has noted, "Baccalaureate education provides a basic understanding of how evidence is developed, including the research process, clinical judgment, interprofessional perspectives, and patient preference as applied to practice."27 Nursing and Respiratory Care have added new guidelines to promote EBP by evaluating published research and establishing evidence-based clinical practice guidelines that are relevant to patient care.

Social workers do the frontline work with the mentally ill population. ${ }^{28}$ However, most social workers do not appear to draw upon research-based practice. ${ }^{29}$ Therefore, on a national level, the social service world is beginning to recognize the significance of EBP in social work practice and explore how evidence-based practice models will help stakeholders find better standards and implement evidence-based approaches. Consistent with the shift in the practice approach, Council on Social Work Education (CSWE) recognizes that teaching social work students how to access, analyze, interpret, and appropriately employ evidence is critical to effective social work practice. ${ }^{30}$ Hence, CSWE incorporated a special provision of EBP in the 2015 Educational Policy and Accreditation Standards (EPAS). Competency 4 - Engage in practice-informed research and research-informed practice stipulates that social workers use practice experience to inform research, employ evidence-based interventions, evaluate their own practice, and use research findings to improve practice, policy, and social service delivery. ${ }^{31}$

Of late, a movement toward EBP in interprofessional curriculum has emerged as there is a great need for healthcare providers to work together to ensure patient safety, quality, and cost-effective care. According to Melnyk we need to, "believe in the value of evidence-based care and have confidence in the ability to implement EBP within a culture that supports it." In this study participants in the focus groups identified barriers that included: "lack of resources, interchanging EBP and research language, quick exposure to EBP and confusion among EBP practices in the clinical setting." Similar findings in other studies ranged from the extreme lag time between research development, dissemination of evidence to practice, and lack of support and training. 5, 21,32 On the other hand, academic courses need to work on information literacy and break the 'silos', thus allowing for the curricular changes necessary to meet student EBP competencies upon graduation. 1,8,13,21

In this study, qualitative and quantitative data revealed participants in all three disciplines recognized the importance of the practice, attitude, and knowledge/skills of EBP. However, it is important to note that there was confusion of the terminology and the word EBP and research was interchanged among most participants during the focus groups. Since the online interprofessional research course included EBP this may have confused students. It was noted by the researchers that the terminology needs to be better explained in the online course. The study showed differences in the survey results between programs. This is likely due to the different clinical exposures and classification levels.

(c) The Internet Journal of Allied Health Sciences and Practice, 2019 
If undergraduate students are educated to adopt the skills that are necessary and modeled to develop an inspired and motivated attitude, the barriers will be reduced in practice.3,8,9 The study revealed that all three programs have been able to provide opportunities for undergraduate students to learn about EBP and recognize its importance to clinical practice. The interprofessional research course has been successful in introducing students to EBP. The study revealed areas in which the faculty can improve the EBP content in the course and individual program curriculums. The most notable area that needs to be addressed is clarifying the difference between EBP and research. In addition, content needs to be adjusted to help students practice critical appraisal and sharing information.

\section{LIMITATIONS}

One limitation of the study is that it is not generalizable due to convenient sample from one university. Another limitation is the selfreporting tool used. It is likely that students participated due to researchers attending class to deliver the survey and those on-line may have been less likely to answer the survey. This was seen in the Respiratory Care online program response rate. The online program results were combined with the on-campus data for increased reliability. Social desirability bias may be present, owning to the pressure that the university has a strong presence of undergraduate research and a required interprofessional undergraduate research course for all health science students. However, the anonymous nature of the survey along with mixed methods approach helped to amend this. Potential biases during focus groups may have been a limitation. Focus groups were conducted by researchers from nursing, respiratory care, and an undergraduate respiratory care student. Prior to the focus group researchers and the undergraduate student discussed the focus group protocol and how to conduct the focus groups. There was a moderator and a researcher in each group. To eliminate bias researchers did not conduct focus groups with students from their discipline. Participants were included in verifying notes taken during the discussion. The fact that students were juniors and seniors may have been another limitation. The differences in perceived Practice, Attitudes, and Knowledge/Skills, among the three programs may be due to the differences in clinical practicums and classification level. Each program has different clinical practicum requirements. Students in each program are exposed to different levels of EBP during clinical courses. Researchers surveyed a large number of students and added a mixed method approach to address this limitation.

\section{CONCLUSION}

This study aimed to assess the practices, attitudes, and knowledge/skills of EBP in health career students in an effort to improve pedagogical practices to fully support learning the EBP process. Results of the data showed that students within the three selected disciplines had a positive attitude (M 5.6) towards EBP. Theme analysis supported this view as participants noted EBP impacts patient safety and quality care. Barriers identified included, "lack of resources, use of research language interchangeably with EBP, quick exposure to EBP and confusion among EBP practices in the clinical setting." Participants had an awareness of EBP and recognized the value of EBP. However, there may be ways to improve the implementation of EBP throughout the learning process while students are in undergraduate studies.

In conclusion, using an interprofessional online course to introduce students to EBP was successful. Further development of additional interprofessional EBP courses or activities should be considered. Health care providers need to use an evidence-based practice approach when making care decisions. EBP knowledgeable practitioners are "able to ask good clinical questions about how and when they should change their practice, demonstrate that they are using good information to base their decisions, evaluate their practice, and know that the outcomes measured are appropriate for the safety and care of their patients." 33

Interprofessional care teams need to work together and use the EBP process in their clinical practice. As a team, they must be able to critically appraise the evidence and evaluate its use in practice and in the development of guidelines and protocols in support of safe quality care. There is a lack of research on the interprofessional approach to teaching EBP in the academic setting to prepare the next generation of health care providers. Results from the study may inform health science educators to respond to areas where students require further support and development of EBP curriculum. This study may encourage others to consider using interprofessional course(s) to introduce health science students to the EBP process.

\section{REFERENCES:}

1. Melnyk, B. M. Breaking down silos and making use of the evidence-based practice competencies in healthcare and academic programs: An urgent call to action. Worldv Evid-Based Nu, 2018; 15(1), 3-4. doi: 10.1111/wvn.12271 
2. Leach, M. J., Hofmeyer, A., \& Bobridge, A. The impact of research education on student nurse attitude, skill and uptake of evidence-based practice: a descriptive longitudinal survey. J Clin Nurs, 2015; 25(1/2), 194203. doi:10.1111/jocn. 13103

3. Linton, M. J., \& Prasun, M. A. Evidence-based practice: collaboration between education and nursing management. J Nurs Manage, 2013; 21(1), 5-16. doi:10.1111/j.1365-2834.2012.01440.x

4. Melnyk, B. M. \& Fineout-Overholt, E. (Eds.) Evidence-based Practice in Nursing and Healthcare: A Guide to Best Practice (3rd ed.). Philadelphia: Lippincott, Williams \& Wilkins.

5. Melnyk, B. M., Gallagher-Ford, L, Zellefrow, C., Tucker, S., Thomas, B., Sinnott, L. T., \& Tan, A. The first U.S. study on nurses' evidence-based practice competencies indicates major deficits that threaten healthcare quality, safety, and patient outcomes. Worldv Evid-Based Nu, 2018; 15(1), 16-25. doi:10.1111/wvn.12269

6. Saunders, H. \& Vehviläinen-Julkunen, K. The state of readiness for evidence-based practice among nurses: An integrative review. Int J Nurs Stud, 2016; 56, 128-140. http://dx/doi/org/10.1016/j.ijnurstu.2015.10.018

7. Scurlock-Evans, L., Upton, P., Rouse, J., \& Upton, D. To embed or not to embed? A longitudinal study exploring the impact of curriculum design on the evidence-based practice profiles of UK pre-registration nursing students. Nurs Educ Today, 2017; 58, 12-18. doi: http://dx.doi.org/10.1016/j.nedt.2017.07.011

8. Ruzafa-Martinez, M., Lopez-Iborra, L., Barranco, D. A., \& Ramos-Morcillo, A. J. Effectiveness of an evidenced-based practice (EBP) course on the EBP competence of undergraduate nursing students: A quasi-experimental study. Nurs Educ Today, 2016; 38, 82-87. doi: http://dxdoi.org/10.1016/j.nedt.2015.12.012

9. van der Zwet, R. M., Kolmer, D. g., \& Schalk, R. Social Workers' Orientation Toward the Evidence-Based Practice Process. Res Social Work Prac, 2016; 26(6), 712-722. doi:10.1177/1049731514540340

10. Bellamy, J. L., Mullen, E. J., Satterfield, J. M., Newhouse, R. P., Ferguson, M., Brownson, R. C., \& Spring, B. Implementing evidence-based practice education in social work: A transdisciplinary approach. Res Social Work Prac 2013; 23(4), 426-436.

11. Knight, C. Social Work Students' Use of the Peer-Reviewed Literature and Engagement in Evidence-Based Practice. J Soc Work Educ, 2015; 51(2), 250-269. doi:10.1080/10437797.2015.1012924

Aglen, B. Pedagogical strategies to teach bachelor students evidence-based practice: A systematic review. Nurs Educ Today, 2016; 36(9), 1188-1189. doi: https://doi.org/10.1016/..nedt.2014.06.001

2. 13. Fiset, V. J., Graham, I. D., \& Davies, B. L. Evidence-based practice in clinical nursing education: A scoping review. J Nurs Educ, 2017; 56(9), 534-541. doi: 10.3928/01484834-20170817-04

3. 14. Parrish, D. E., \& Rubin, A. Social workers' orientations toward the evidence-based practice process: A comparison with psychologists and licensed marriage and family therapists. Social Work, 2012; 57, 201-

210. doi.org/10.1093/sw/sws016.

4. 15. Heiwe, S, Kajermo, K, Tyni-Lenné, R, et al. Evidence-based practice: attitudes, knowledge and behaviour among allied health care professionals Int J Qual Health Care,2011; 23(2), 198-209.

5. 16. Penz, K. \& Bassendowski, S. Evidence-based nursing in clinical practice: Implications for nurse educators. J Contin Educ Nurs, 2006; 37(6), 250-254. Doi:org/10.3928/0022012420061101-03.

6. 17. Maben, J., Latter, S. \& Macleod-Clark, J. The theory-practice gap: impact of professional-bureaucratic work conflict on newly-qualified nurses. J Adv Nurs, 2006; 55(4), 465-477. http://dx.doi.org/10.1111/j.1365-2648.2006.03939.x.

7. 18. Mooney, M. Professional socialization: the key to survival as a newly qualified nurse. Int J Nurs Pract, 2007; 13(2), 75-80. doi: 10.1111/j.1440-172X.2007.00617.x

8. 19. Tucker, B., Jones, S., Mandy, A. \& Gupta, R. Physiotherapy students' sources of stress, perceived course difficulty and paid employment: comparison between Western Australia and United Kingdom. Physiother Theor Pr, 2006; 22(6), 317-328. http://dx.doi.org/10.1080/09593980601059550

9. 20. Banks, P., Roxburgh, M., Kane, H., et al (2011). Flying Start NHSTM: Easing the transition from student to registered health professional. J Clin Nurs, 20 (23-24). doi: 3567-3576. 10.1111/j.1365-2702.2011.03796.x

10. 21. Melnyk, B. M., Gallagher-Ford, L., Thomas, B. K., Troseth, M., Wyngarden, K., \& Szalacha, L. A study of chief nurse executives indicates low prioritization of evidence-based practice and shortcomings in hospital performance metrics across the United States. Worldv Evid-Based Nu, 2016; 13(1), 6-14. doi: 10.1111/wvn.12133

11. 22. Upton, P., Scurlock-Evans, L., \& Upton, D. Development of the student evidence-based practice questionnaire (SEBPQ). Nurs Educ Today, 2016; 37, 38-44. http://dx.doi.org/10.1016/j.nedt.2015.11.010

12. 23. Beccaria, L., Beccaria, G., \& McCosker, C. A confirmatory factor analysis of the student evidence-based practice questionnaire (s-ebpq) in an Australian sample. Nurs Educ Today, 2018; 62, 69-73. doi.org/10.1016/..nedt.2017.12.010

13. 24. Committee on Facilitating Interdisciplinary Research and Committee on Science $\mathrm{E}$, and Public Policy. Facilitating Interdisciplinary Research. Washington, DC: National Academies of Science, 200

14. 25. Guidelines for Respiratory Care Department Protocol Program Structure; 2008. Retrieved from http://www.aarc.org/resources/professional-documents/whitepapers/protocol-program-structure/ 
15. 26. Barnes, T. A., Gale, D. D., Kacmarek, R. M., \& Kageler, W. V. Competencies needed by graduate respiratory therapists in 2015 and beyond. Resp Care, 2010; 55(5), 601-616.

16. 27. Association of Colleges of Nursing (AANC); 2008. The essentials of baccalaureate education for professional nursing practice. Washington D.C.: AACN.

17. 28. Insel, T. Science to service: Mental health care after the decade of the brain. Presentation given at Society of Social Work Research Annual Conference, 2014; New Orleans, Louisiana.

18. 29. Gibbs, L., \& Gambrill, E. Evidence-based practice: Counter-arguments to objections. Res Social Work Prac, 2002; $12,452-476$.

19. 30. https://www.cswe.org/Centers-Initiatives/Initiatives/Teaching-Evidence-Based-Practice

20. 31. Council on Social Work Education (CSWE) - EPAS Handbook, 2015 https://www.cswe.org/Accreditation/Standards-and-Policies/EPAS-Handbook

21. 32. Bellamy, J.L., Bledsoe, S.E., and Traube, D.E. Current State of Evidence-Based Practice in Social Work: A Review of the Literature and Qualitative Analysis of Expert Interviews. J Evid-Based Social Work, 2006; 3(1): 23 48. doi.org/10.1300/j394v03n01_02

22. 33. International Council of Nurses [ICN], 2012. Closing the gap: From evidence to action. Geneva: Switzerland. Retrieved from https://www.nursingworld.org/ 4aff6a/globalassets/practiceandpolicy/innovation--evidence/ind-kit-2012for-nnas.pdf

\section{APPENDIX A}

\section{General Introduction:}

\section{Protocol for Focus Groups}

Thank you for your willingness to participate in this study. The focus group discussion should take approximately one hour. There are several questions I have prepared for this study. I may ask additional questions for clarifications such as, "Can you expand on that issue?" or "How did that make you feel?" If you are uncomfortable with any of the questions I ask, please let me know immediately and I will move to the next question. You may choose to leave at any time. Please understand that your identity will be kept confidential, and your responses to any of the questions will not be reported in a way that could reveal who you are. Participation is voluntary, you can quit at any time, and there is no effect upon you grades or relationship with your program and/or the university. Do you have any questions before we begin?

I would like to ask you some questions about your practice, attitude, and knowledge/skills with EBP. We will collect themes from each question and list these on the flip chart.

1. As a student, how did you learn about EBP?

2. Can you describe how you are utilizing any part of the EBP process in your studies?

3. Why is EBP important for a practitioner to know about EBP?

4. One factor that was most important to you as you learned about EBP.

As we conclude this focus group, is there any theme listed for a question that you would like to explore and/or elaborate on?

\section{Concluding Remarks:}

Thank you again, for your time and willingness to participate in this study. 